\title{
DETERMINISTIC-STOCHASTIC BOUNDARY ELEMENT MODELING OF THE BRAIN AND EYE EXPOSED TO HIGH-FREQUENCY RADIATION
}

\author{
D. POLJAK ${ }^{1}$, H. DODIG ${ }^{2}$, M.CVETKOVIĆ ${ }^{1}$, S. LALLECHERE $^{3}$, K. EL KHAMLICHI DRISSI ${ }^{3} \&$ P.BONNET $^{3}$ \\ ${ }^{1}$ University of Split, Split, Croatia. \\ ${ }^{2}$ Marine Center for Electronics, Split, Croatia. \\ ${ }^{3}$ Blaise Pascal University, Clermont-Ferrand, France.
}

\begin{abstract}
The paper reviews the influence of the variability in the morphology and the tissue properties of the human brain and eye, respectively, exposed to high-frequency (HF) radiation. Deterministic-stochastic modeling enables one to estimate the effects of the parameter uncertainties on the maximum induced electric field and Specific Absorption Rate (SAR). Surface Integral Equation (SIE) scheme applied to the brain exposed to HF radiation and hybrid boundary element method (BEM)/finite element method (FEM) scheme used to handle the eye exposure to HF radiation are discussed.

Furthermore, a simple stochastic collocation (SC), through which the relevant parameter uncertainties are taken into account, is presented. The SC approach also provides the assessment of corresponding confidence intervals in the set of obtained numerical results. The expansion of statistical output in terms of the mean and variance over a polynomial basis (via $\mathrm{SC}$ approach) is shown to be robust and efficient method providing a satisfactory convergence rate. Some illustrative numerical results for the maximum induced field and SAR in the brain and eye, respectively, are given in the paper, as well.

Keywords: boundary integral equations, deterministic modeling, human exposure to electromagnetic fields, stochastic modeling.
\end{abstract}

\section{INTRODUCTION}

Human exposure to artificial electromagnetic fields has raised many questions regarding potential adverse effects [1], particularly for the brain and eye exposure to high-frequency (HF) radiation. The assessment of HF exposure is based on the evaluation of specific absorption rate (SAR) distribution and related temperature rise in a tissue. As a measurement of fields induced in the body is not possible, human exposure assessment is carried out via sophisticated computational models [2-5]. Contrary to the simple canonical models used in the 60 s and 70s (plane slab, cylinders, homogeneous and layered spheres and prolate spheroids [6]) modern realistic, anatomically based computational models comprising cubical cells are mostly related to the use of the Finite Difference Time Domain (FDTD) methods [7]. The Finite Element Method (FEM) and Boundary Element Method (BEM) are generally used to a somewhat lesser extent $[3,8]$.

One of the significant difficulties arising in the area of computational bioelectromagnetics is the appreciable variation of the input parameter set, i.e. possible differences in individual size and age (morphology), or the general variability of permittivity and conductivity, due to difference in age or sex. The uncertainty of the input parameters set eventually leads to the 
uncertainty of dosimetric model outputs such as induced electric field and SAR. Thus, one of the key challenges that numerical dosimetry faces today is the quantification and the treatment of these uncertainties.

A novel approach to tackle this problem is to use so called stochastic dosimetry [9, 10], combining deterministic electromagnetic techniques with certain statistical methods.

The present paper deals with the influence of the variability in the morphology and the tissue properties of the brain and eye, respectively, to the related SAR due to the exposure to $\mathrm{HF}$ electromagnetic fields. Stochastic-deterministic modeling provides a satisfactory theoretical basis for estimating the effects of the corresponding uncertainties on the maximum induced local and average SAR, respectively. An efficient BEM scheme to treat the brain exposure and hybrid BEM/finite element method (FEM) used to handle the eye exposure are considered. Having completed the deterministic modeling a simple stochastic collocation (SC) formalism is applied to accurately account for uncertainties and to assess confidence intervals in the set of obtained numerical results.

\section{FORMULATION: DETERMINISTIC AND STOCHASTIC APPROACH}

The main task of HF dosimetry is to quantify thermal effects, i.e. to assess the level and distribution of the electromagnetic energy absorbed by the body. The main dosimetric quantity for quantifying the effects of HF radiation is the SAR and related temperature increase.

\subsection{Specific absorption rate}

The $S A R$, a fundamental quantity in HF dosimetry, is defined in terms of the rate of energy $W$ absorbed by, or dissipated in the unit body mass $m$ :

$$
S A R=\frac{d p}{d m}=\frac{\sigma}{2 \rho}\left|E^{i n d}\right|^{2} .
$$

where $P$ is the dissipated power, $E^{\text {ind }}$ is the peak value of the electric field induced inside a tissue, $\rho$ is the tissue density and $s$ is the tissue conductivity.

In addition to incident field parameters, the absorbing and reflecting effects of the environment, as well as the properties of the exposed body, also significantly influence the SAR distribution. Maximal values of $S A R$ in the body are induced when the electric field is oriented parallel to the longitudinal body axis.

\subsection{Deterministic modeling}

The lossy dielectric model of the brain exposed to HF electromagnetic fields is based on the surface integral equation (SIE) formulation which could be derived from the equivalence theorem and by forcing the corresponding interface conditions for the electric and/or magnetic field, [11, 12] as indicated in Fig. 1.

The lossy homogeneous object representing the brain is excited by the incident electromagnetic field characterized by $\vec{E}^{i n c}$ and $\vec{H}^{i n c}$.

Performing some mathematical manipulations, the following set of coupled SIEs is obtained $[11,12]$ : 


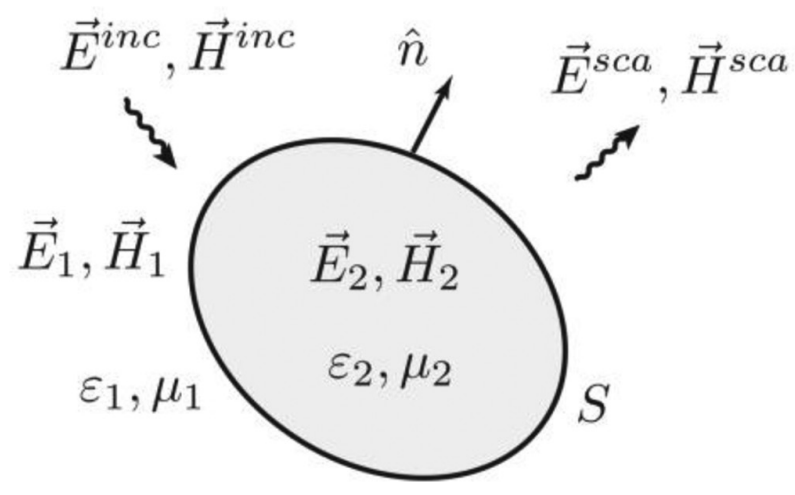

Figure 1: The lossy homogeneous dielectric brain model.

$$
\begin{aligned}
& j \omega \mu_{n} \iint_{S} \vec{J}\left(\vec{r}^{\prime}\right) G_{n}\left(\vec{r}, \vec{r}^{\prime}\right) \mathrm{d} S^{\prime}- \\
& -\frac{j}{\omega \varepsilon_{n}} \iint_{S} \nabla_{S}^{\prime} \cdot \vec{J}\left(\vec{r}^{\prime}\right) \nabla G_{n}\left(\vec{r}, \vec{r}^{\prime}\right) \mathrm{d} S^{\prime}+ \\
& +\iint_{S} \vec{M}\left(\vec{r}^{\prime}\right) \times \nabla^{\prime} G_{n}\left(\vec{r}, \vec{r}^{\prime}\right) \mathrm{d} S^{\prime}= \begin{cases}\vec{E}^{i n c}, & n=1 \\
0, & n=2 .\end{cases}
\end{aligned}
$$

where $E^{i n c}$ stands for the incident electric field illuminating the biological tissue, $\vec{J}$ and $\vec{M}$ are equivalent electric and magnetic current density, respectively, $G_{\mathrm{n}}$ is the interior/exterior Green function given by [11]:

$$
G_{n}\left(\vec{r}, \vec{r}^{\prime}\right)=\frac{e^{-j k_{n} R}}{4 \pi R} ; \quad R=\left|\vec{r}-\vec{r}^{\prime}\right|
$$

and $R$ is the distance from the source to observation point, respectively, while $k_{\mathrm{n}}$ denotes the wave number of a medium $n$.

The set of integral equations (2) is solved by means of an efficient BEM scheme reported in $[11,12]$.

Plane wave incidence on the corneal part of the eye representing an unbounded scattering problem can be formulated via the Stratton-Chu formulation, i.e. the time-harmonic electric field in the exterior domain is expressed by the following boundary integral equation [3]:

$$
\alpha \vec{E}=\vec{E}_{i}+\oint_{\partial V^{\prime}} \vec{n}^{\prime} \times\left(\nabla^{\prime} \times \vec{E}\right) G d S^{\prime}+\oint_{\partial V^{\prime}}\left[\left(\vec{n}^{\prime} \times \vec{E}\right) \times \nabla^{\prime} G+\left(\vec{n}^{\prime} \cdot \vec{E}\right) G\right] d S^{\prime}
$$

where $E_{\mathrm{i}}$ stands for the incident electric field, $\vec{n}$ is an outer normal to surface $\partial V^{\prime}$. bounding the volume $V$ representing the eye and $\alpha$ is the solid angle subtended at an observation point, while $G$ represents the fundamental solution of the corresponding Helmholtz equation [9].

Mathematical details for hybrid BEM/FEM are available in $[3,13]$. 


\subsection{Stochastic analysis}

Once the deterministic modeling via BEM and BEM/FEM, respectively is carried out a stochastic post-processing of the obtained numerical results arising from the deterministic model can be performed via stochastic Collocation (SC) technique by simply choosing one or more random variables depending on the problem of interest. It is worth noting that stochastic analysis is found to be rather useful in many cases with various uncertainties in the input data set, such as wire structures in complex environment, grounding systems in a rocky terrain, ground penetrating radar (GPR) or particularly in bioelectromagnetics. The need for stochastic analysis is quite obvious in the case of bioelectromagnetics as one hardly could determine the conductivity and/or dielectric constant of the brain or eye precisely.

The fundamental principle of SC technique is to use the polynomial approximation of the output $E$ of interest for $\mathrm{N}$ given random parameters. The random parameter $\mathrm{Z}$ is defined as $[9,10]$ :

$$
Z=Z^{0}+\hat{\mathrm{u}}
$$

where $Z^{0}$ is the initial (mean) value, while $\hat{\mathrm{u}}$ is the random variable (RV) with the assigned statistical distribution.

The function $t \rightarrow E\left(Z^{0}, t\right)$ is expanded over a stochastic space using the Lagrangian basis functions set $[9,10]$ :

$$
E\left(Z^{0}, t\right)=\sum_{i=1}^{n} E_{i}\left(Z^{0}\right) L_{i}(t)
$$

where $E\left(Z^{0}, t\right)$ is the output of interest (electric field in this work), while Lagrange polynomial $L_{\mathrm{i}}(\mathrm{t})$ are defined, as follows:

$$
L_{i}(t)=\prod_{j=1, j \neq i}^{n} \frac{\left(t-t_{j}\right)}{\left(t_{j}-t_{i}\right)}
$$

Exploiting the property of the Lagrangian basis, yields:

$$
E\left(Z^{0}, t_{i}\right)=E_{i}\left(Z^{0}\right)
$$

According to the statistical definition for the mean $\mu$ [7],

$$
\mu=\left\langle E\left(Z^{0}, t\right)\right\rangle=\int_{D} E\left(Z^{0}, u\right) p(u) d u
$$

where $p(u)$ denotes the probability density function of RV û from (6) and $D$ denotes the random variable domain, the expected value $<>$ of the considered output $E$ taking into account (6) and (8) can be written, as follows:

$$
\left\langle E\left(Z^{o}, t\right)\right\rangle=\sum_{i=1}^{n} E_{i}\left(Z^{0}\right) \int_{D} L_{i}(u) p(u) d u
$$


Expression (10) can be written in the form:

$$
\left\langle E\left(Z^{0}, t\right)\right\rangle=\sum_{i=1}^{n} E_{i}\left(Z^{0}\right) w_{i}
$$

where $w_{\mathrm{i}}$ is given by integral:

$$
w_{i}=\int_{D} L_{i}(u) p(u) d u
$$

Furthermore, according to statistical definition the variance $\sigma_{\mathrm{var}}^{2}$ is defined by following expression:

$$
\sigma_{\mathrm{var}}^{2}=\int_{D}\left[E\left(Z^{0}, u\right)-\left\langle E\left(Z^{0}, t\right)\right\rangle\right]^{2} p(u) d u
$$

which, after performing some mathematical manipulations, can be written, as follows:

$$
\sigma_{\mathrm{var}}^{2}=\sum_{i=0}^{n} w_{i} E_{i}^{2}\left(Z^{0}\right)-\left(\sum_{i=0}^{n} w_{i} E_{i}\left(Z^{0}\right)\right)^{2}
$$

The order (n-1) of approximation, i.e. the convergence, depends on the number of chosen collocation points. The computation of integral (12) is based on Gaussian quadrature.

The stochastic analysis is presented for the case of single RV but it can be easily extended to the case of $\mathrm{N} \mathrm{RVs}[9,10]$. Also, in addition to the mean, other higher statistical moments can be readily determined for the case of one-dimensional and multidimensional RV case [10].

Further mathematical details for SC procedures can be found elsewhere, e.g. in $[9,10]$.

\section{COMPUTATIONAL EXAMPLES}

The first set of Figures is related to the results obtained from deterministic brain modeling.

Figures 2 and 3 show the $S A R$ distribution in the brain at $f=900 \mathrm{MHz}$ and $f=1,800 \mathrm{MHz}$, respectively, for the case of vertical polarization. The power density of the incident plane wave is $P=5 \mathrm{~mW} / \mathrm{cm}^{2}$. The brain parameters of interest are presented in Table 1 .

The solution is carried out via the BEM by discretizing the brain to 696 triangular elements and 1,044 edge-elements.

The obtained peak and average $S A R$ values for vertical polarization at $f=900 \mathrm{MHz}$ and $f=1,800 \mathrm{MHz}$ are given in Table 2 .

The obtained peak $S A R$ values in the brain do not exceed the ICNIRP limits [14] for localized $S A R$ in the head averaged per $10 \mathrm{~g}$ of tissue ( $10 \mathrm{~W} / \mathrm{kg}$ for the occupational exposure).

However, the exposure limit for the general public exposure limit $(2 \mathrm{~W} / \mathrm{kg}$ localized in the head and trunk) has been exceeded at $f=1,800 \mathrm{MHz}$.

Fig. 4 shows the SC convergence with 3, 5 and 7 points for the SAR value calculations and different random variables $\left(\mathrm{RV}_{\mathrm{k}} ; \mathrm{k}=1, \ldots, 5\right)$.

The dimensions of the average adult human brain are, as follows: width $131.8 \mathrm{~mm}$, length $161.1 \mathrm{~mm}$, height $139 \mathrm{~mm}$, while the frequency dependent parameters of the human brain are discussed in [12]. Furthermore, the value for the relative permittivity and the electrical conductivity of the brain are: $\varepsilon_{\mathrm{r}}=45.805$ and $\sigma=0.766 \mathrm{~S} / \mathrm{m}$, respectively. Each RVk 
SAR $[\mathrm{W} / \mathrm{kg}]$
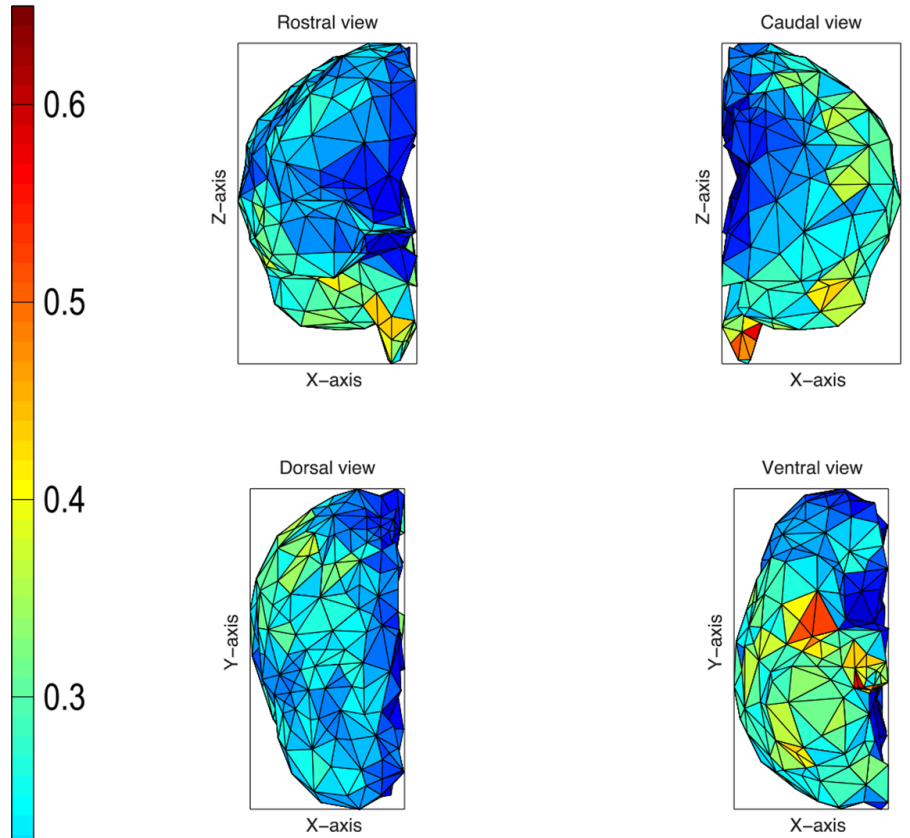

0.2
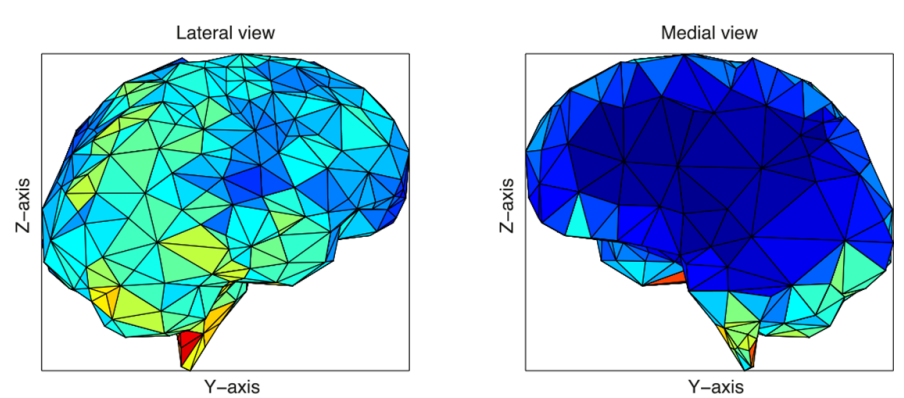

Figure 2: SAR distribution at $f=900 \mathrm{MHz}$.

Table 1: The brain electrical parameters.

\begin{tabular}{lll}
\hline & $f=900 \mathrm{MHz}$ & $f=1,800 \mathrm{MHz}$ \\
\hline$\varepsilon_{\mathrm{r}}$ & 46 & 84 \\
$\sigma[\mathrm{S} / \mathrm{m}]$ & 0.8 & 1.2 \\
\hline
\end{tabular}

Table 2: The peak and average $S A R$ values for different exposure scenarios.

\begin{tabular}{lll}
\hline & $f=900 \mathrm{MHz}$ & $f=1,800 \mathrm{MHz}$ \\
\hline$S A R_{\max }[\mathrm{W} / \mathrm{kg}]$ & 0.866 & 2.678 \\
$S A R_{\mathrm{avg}}[\mathrm{W} / \mathrm{kg}]$ & 0.158 & 0.348 \\
\hline
\end{tabular}



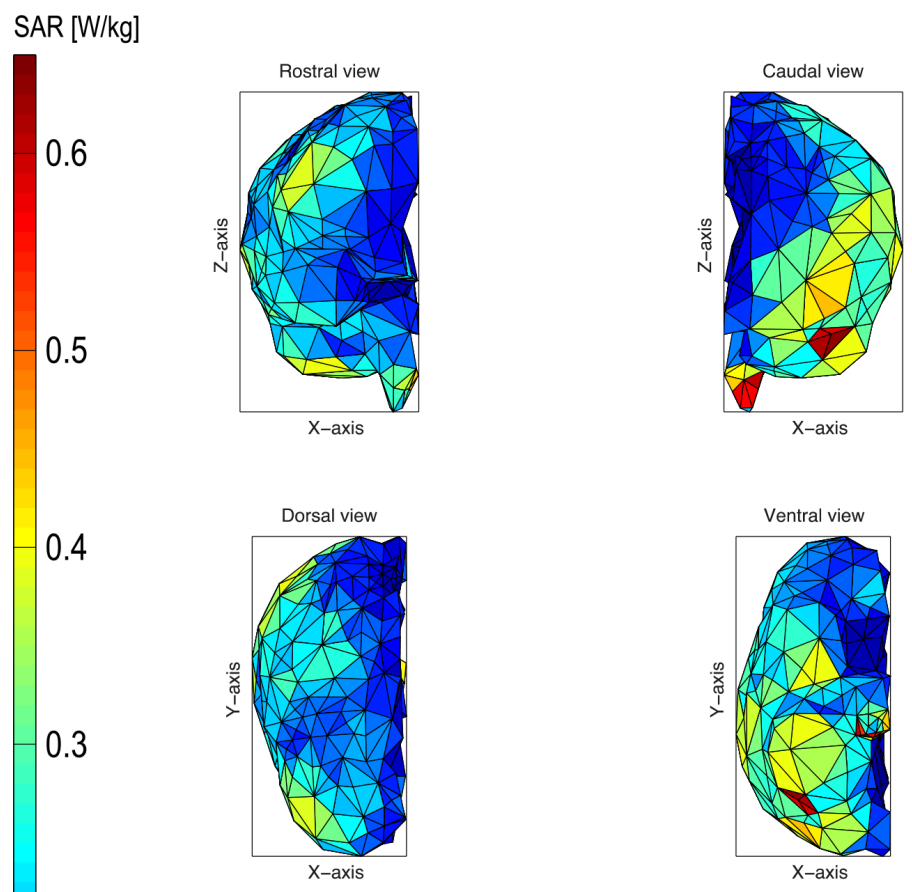

0.2
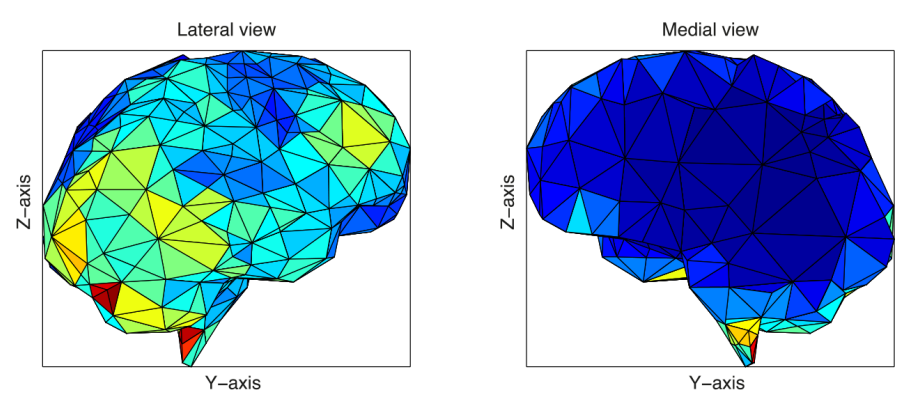

Figure 3: $S A R$ distribution at $f=1,800 \mathrm{MHz}$.

$(\mathrm{k}=1, \ldots, 5)$ is assumed to be uniformly distributed around deterministic values with common coefficient of variation equal to $5.77 \%$ [12].

Although the output (maximum SAR) is highly non linear, SC provides an assessment of the 1st statistical moment with 5 simulations only. Note that several univariate calculations, i.e. calculations for a single random variable, are carried out.

The following set of Figures is related to the results obtained from deterministic and stochastic eye modeling.

Figure 5 shows the $S A R$ distribution in the eye due to the exposure to plane wave with power density of $10 \mathrm{~W} / \mathrm{m}^{2}$ obtained by the use of hybrid BEM/FEM. The eye parameters are available elsewhere, e.g. in [13]. Figure 6 gives information of the first-order sensitivity of the model to a corresponding random variable for the SAR assessment in the eye exposed to plane wave, i.e. the SAR variance. 


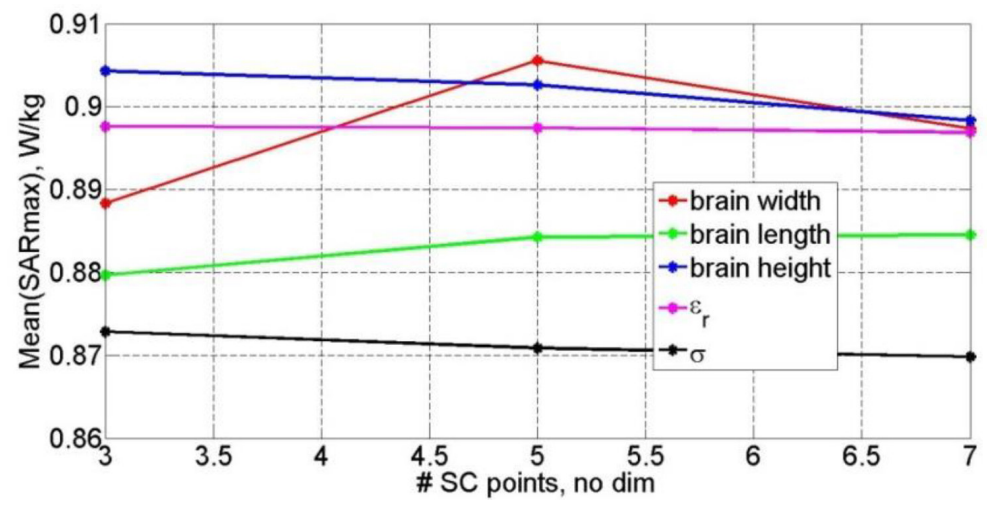

Figure 4: Mean of maximum SAR.

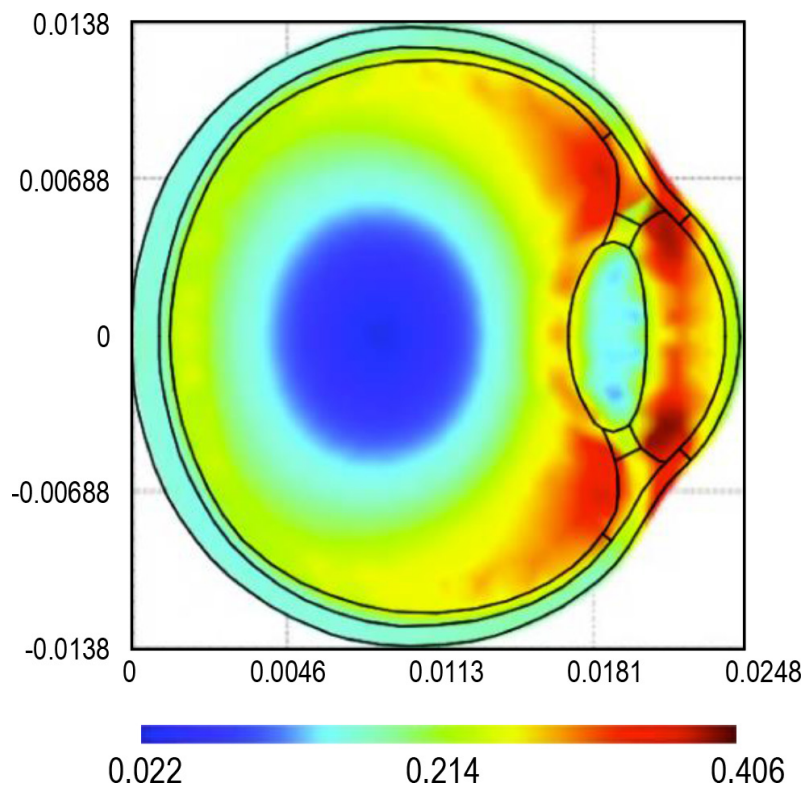

Figure 5: SAR in the eye due to plane wave exposure $10 \mathrm{~W} / \mathrm{m}^{2}$ at $f=1 \mathrm{GHz}$.

The conductivity of the vitreous body is $\sigma_{v}^{0}=0.70112 \mathrm{~S} / \mathrm{m}$ at $f=6 \mathrm{GHz}$ and is assumed to be uniformly distributed around interval $\sigma_{v}=\sigma_{v}^{0} \pm 0.6 \mathrm{~S} / \mathrm{m}$.

As depicted in Fig. 6, higher levels of $\sigma_{S A R}$ are concentrated inside the vitreous body.

\section{CONCLUDING REMARKS}

The paper presents deterministic-stochastic analysis of the exposure of the brain and eyes to the HF radiation, thus taking into account the uncertainty variations of the several input parameters. The deterministic model is based on the corresponding boundary integral equa- 

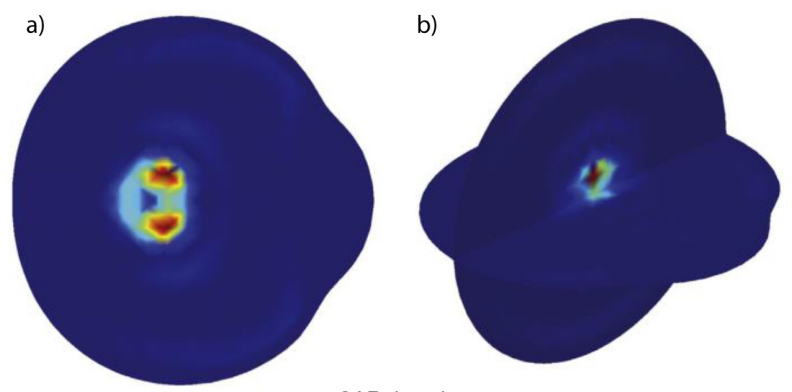

SAR_Levelset

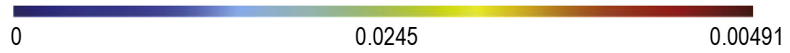

Figure 6: $\sigma_{\mathrm{SAR}}$ inside the eye (stochastic variations around vitreous body conductivity) induced by plane wave with power density $10 \mathrm{~W} / \mathrm{m}^{2}$ at $f=6 \mathrm{GHz}$ and $\mathrm{SC}$ approximation order $n=2$ : (a) z-plane; (b) x-plane and y-plane.

tion formulation and related solution methods based on the BEM scheme (the brain exposure), and hybrid BEM/finite element method (BEM/FEM) scheme (the eye exposure). The expansion of the statistical output in terms of the mean and variance over a polynomial basis via stochastic collocation (SC) is shown to be robust and efficient technique providing a satisfactory convergence rate of the $\mathrm{SC}$ technique.

\section{REFERENCES}

[1] Poljak, D., Electromagnetic fields: environmental exposure. Encyclopedia of Environmental Health, ed, J.O. Nriagu, Elsevier: Burlington, pp. 259-268, 2011. http://dx.doi.org/10.1016/B978-0-444-52272-6.00422-0

[2] Hand, J.W., Modeling the interaction of electromagnetic fields (10 MHz-10 GHz) with the human body: methods and applications. Physics in Medicine and Biology, 53(16), pp. 243-286, 2008. http://dx.doi.org/10.1088/0031-9155/53/16/R01

[3] Poljak, D., Čavka, D., Dodig, H., Peratta, C. \& Peratta, A., On the use of boundary element analysis in bioelectromagnetics. Engineering Analysis with Boundary Elements, (Special issue on Bioelectromagnetics), 49, pp. 2-14, 2014.

[4] Singh, K.D., Longan, N.S. \& Gilmartin, B., Three dimensional modeling of the human eye based on magnetic resonance imaging. Investigative Opthamology and Visual Science, 47, pp. 2272-2279, 2006. http://dx.doi.org/10.1167/iovs.05-0856

[5] Hirata, A., Temperature increase in human eyes due to near-field and far-field exposures at $900 \mathrm{MHz}, 1.5 \mathrm{GHz}$, and $1.9 \mathrm{GHz}$. IEEE Transactions on Electromagnetic Compatibility, 47(1), pp. 68-76, 2005. http://dx.doi.org/10.1109/TEMC.2004.842113

[6] Poljak, D., Human Exposure to Electromagnetic Fields, WIT Press: SouthamptonBoston, 2003.

[7] Fujimoto, M., Hirata, A., Wang, J., Fujiwara, O. \& Shiozawa, T., FDTD-derived correlation of maximum temperature increase and peak SAR in child and adult head models 
due to dipole antenna. IEEE Transactions on Electromagnetic Compatibility, 48(1), pp. 240-247, 2006.

[8] Poljak, D., Dodig, H., Čavka, D. \& Peratta, A., Some numerical methods of thermal dosimetry for applications in bioelectromagnetics. Proceedings of Heat Transfer 2012, Split, Croatia, pp. 271-280, 2012.

http://dx.doi.org/10.2495/ht120231

[9] Dodig, H., Lallechere, S., Bonnet, P., Poljak, D. \& El Khamlichi Drissi, K., Stochastic sensitivity of the electromagnetic distributions inside a human eye modeled with a 3D hybrid BEM/FEM edge element method. Engineering Analysis with Boundary Elements, 49, pp. 48-62, 2014.

http://dx.doi.org/10.1016/j.enganabound.2014.04.005

[10] Cvetković, M., Lallechere, S., El Khamlichi Drissi, K., Bonnet, P. \& Poljak, D., Stochastic sensitivity in homogeneous electromagnetic-thermal model of human brain. Workshop on Uncertainty Modeling for ElectroMagnetic Applications UMEMA2015, Clermont-Ferrand, France, 2015.

[11] Cvetković, M. \& Poljak, D., An efficient integral equation based dosimetry model of the human brain. Proceedings of the 2014 International Symposium on Electromagnetic Compatibility (EMC Europe 2014), Gothenburg, Sweden,2014, pp. 375-380, 2014. http://dx.doi.org/10.1109/emceurope.2014.6930935

[12] Cvetković, M., Poljak, D. \& Hirata, A., The electromagnetic-thermal dosimetry for the homogeneous human brain model. Engineering Analysis with Boundary Elements. 63, pp. 61-73, 2016.

http://dx.doi.org/10.1016/j.enganabound.2015.11.002

[13] Dodig, H., Poljak, D. \& Peratta, A., Hybrid BEM/FEM edge element computation of the thermal rise in the 3D model of the human eye induced by high frequency EM waves. 2012 International Conference on Software, Telecommunications and Computer Networks, Split, pp. 1-5, 2012.

[14] International Commission on Non-Ionizing Radiation Protection, Guidelines for limiting exposure to time-varying electric, magnetic and electromagnetic fields (up to 300 GHZ). Health Physics, 74(4), pp. 494-522, 1998. 